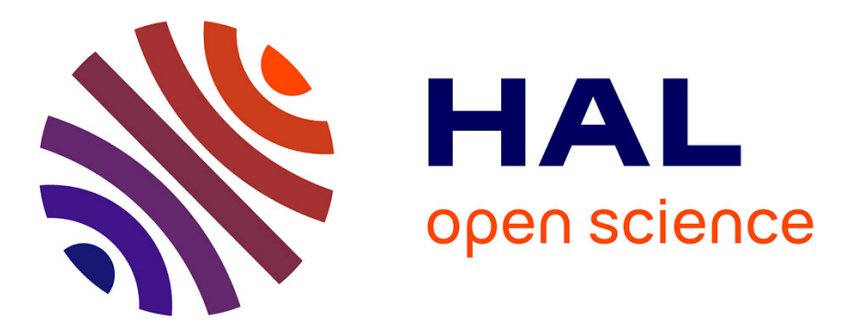

\title{
Porous diamond foam with nanometric diamond grains using Bias Enhanced Nucleation on iridium
}

\author{
N. Vaissiere, Samuel Saada, K.H. Lee, M. Mermoux, Mohamed Sennour, \\ Jean-Charles Arnault
}

\section{- To cite this version:}

N. Vaissiere, Samuel Saada, K.H. Lee, M. Mermoux, Mohamed Sennour, et al.. Porous diamond foam with nanometric diamond grains using Bias Enhanced Nucleation on iridium. Diamond and Related Materials, 2016, 68, pp.23-27. 10.1016/j.diamond.2016.04.009 . hal-01340054

HAL Id: hal-01340054

https://hal-mines-paristech.archives-ouvertes.fr/hal-01340054

Submitted on 25 Mar 2020

HAL is a multi-disciplinary open access archive for the deposit and dissemination of scientific research documents, whether they are published or not. The documents may come from teaching and research institutions in France or abroad, or from public or private research centers.
L'archive ouverte pluridisciplinaire HAL, est destinée au dépôt et à la diffusion de documents scientifiques de niveau recherche, publiés ou non, émanant des établissements d'enseignement et de recherche français ou étrangers, des laboratoires publics ou privés. 
Porous diamond foam with nanometric diamond grains using Bias Enhanced Nucleation

\author{
N. Vaissiere ${ }^{1}$, S. Saada ${ }^{1}$, K. H. Lee ${ }^{1}$, M. Mermoux ${ }^{2,3}$, M. Sennour ${ }^{4}$, J. C. Arnault ${ }^{*}$ \\ ${ }^{1}$ CEA, LIST, Diamond Sensors Laboratory, F-91191 Gif sur Yvette, France. \\ ${ }^{2}$ Univ. Grenoble Alpes, LEPMI, F-38000 Grenoble, France. \\ ${ }^{3}$ CNRS, LEPMI, F-38000 Grenoble, France. \\ ${ }^{4}$ Centre des Matériaux, Mines ParisTech CNRS UMR 7633, BP 87, F-91003 Evry Cedex, \\ France. \\ *corresponding author \\ +33 (0)1 69087102 / (jean-charles.arnault@cea.fr, J-C. Arnault)
}

\begin{abstract}
A porous diamond foam exhibiting a three-dimensional structure were synthesized by applying prolonged Bias Enhanced Nucleation on iridium buffer layers. The Raman spectrum of this material recorded at $325 \mathrm{~nm}$ looks very close to the signature obtained for detonation nanodiamonds. HRTEM investigations well confirm the presence of diamond crystals with nanometric size between 2 and $6 \mathrm{~nm}$. These aggregated diamond nanocrystals form a threedimensional structure very different from ultrananocrystalline diamond (UNCD) generally embedded in a graphitic matrix. According to its high specific surface area, such a 3D material may have promising applications for sensors.
\end{abstract}

Keywords: BEN; three-dimensional structure; porous diamond foam; HRTEM; Raman 
Several previous attempts were reported to fabricate diamond materials with enhanced specific surface area. Indeed, it is a great challenge to use diamond for sensors applications. For example, Masuda et al. used a porous alumina mask to fabricate a diamond matrix by oxygen etching [1]. Another approach combines a graphitization of a boron doped diamond (BDD) film with an oxygen etching leading to a porous diamond material [2]. The seeding of templates with nanodiamonds was also used as a powerful technique to obtain diamond foam. $\mathrm{SiO}_{2}$ spheres were seeded with detonation nanodiamonds and a BDD coating was overgrown $[3,4]$. After removal of $\mathrm{SiO}_{2}$ core by $\mathrm{HF}$ etching, diamond foam was used as an electrode. The growth of BDD porous membranes usable for peptides detection was also achieved by seeding of fiberglass filters [5]. An alternative way to obtain diamond membranes is the chemical etching of nanocrystalline diamond / 3C-SiC composites [6]. Recently, Hébert et al. reported that properties of electrodes combining carbon nanotubes and BDD are governed by their high aspect ratio [7].

The present letter focuses on the synthesis of a diamond porous material composed of nanometric crystals using prolonged Bias Enhanced Nucleation (BEN) on iridium buffer layers. Up to now, BEN is effectively the most powerful procedure to induce diamond heteroepitaxy on substrates of different natures [8]. According to its specific surface reactivity to CVD environment, iridium is currently the best heterosubstrate to obtain highly oriented diamond films. Using specific BEN conditions, a porous diamond foam can also be grown originating from islands at iridium surface (Figure 1). The crystalline structure as well as the chemistry of this carbon material was investigated using High Resolution Transmission Electron Microscopy (HRTEM) and Raman spectroscopy.

Iridium buffer layers were epitaxially grown on $\mathrm{SrTiO}_{3}$ (001) substrates using an evaporation cell [9]. Their polar and azimuthal misorientations measured by X-ray diffraction are $0.22^{\circ}$ and $0.26^{\circ}$, respectively. BEN process includes three different steps: a surface cleaning under pure hydrogen plasma with a MW power/pressure couple of 400W/20mbar during $10 \mathrm{~min}$, a stabilization of the substrate temperature by adding $4 \%$ of methane in hydrogen plasma before the BEN step during $10 \mathrm{~min}$. Details about BEN conditions such as how to apply the bias voltage and measure the bias current on $\mathrm{Ir} / \mathrm{SrTiO}_{3}$ were elsewhere reported [9]. Briefly, the substrate holder was electrically insulated from the metallic walls of the reactor, which were maintained at ground potential; thus, it was possible to apply a negative voltage to the 
substrate holder during plasma exposition. The iridium surface underwent a BEN step at -307 $\mathrm{V}$ corresponding to a bias current of $17 \mathrm{~mA}$ and a temperature of $660^{\circ} \mathrm{C}$. No further CVD growth step was applied. Field Emission Scanning Electron Microscopy (FE-SEM) revealed a very different surface morphology compared to the one leading to heteroepitaxy or UNCD films. Figure 1Figure 1 shows the formation of the 3D structure after 20 minutes of BEN step. It originates from islands at iridium surface and forms a large covering of the substrate at longer time. Any crystals shape is observable using the in lens detector of FE-SEM allowing to reach a high lateral resolution close to $3 \mathrm{~nm}$. According to the cross-section provided on Figure 2Figure $2 \mathrm{c}$, the growth rate is estimated to $4 \mu \mathrm{m} / \mathrm{h}$. This cross-section well exhibits a microstructure very different compared to NanoCrystalline Diamond (NCD) film grown using the same CVD reactor (Figure 2Figure $2 \mathrm{~d}$ ). The porous character of the diamond material can be evidenced by indentation experiments. In fact, micro-indentation leads to an irreversible deformation without crack formation (Figure 2a) of the film compared to surrounding areas which is an evidence of the presence of voids. The same indentation procedure applied to NCD film does not induce any residual deformation; we only observed metal deposition (dark traces) on the contact area (Figure $2 b$ ). These two materials have totally different behavior under micro-indentation because of their structures: NCD film is composed of nanodiamond crystals without void whereas the structure obtained by prolonged bias on iridium induces a film composed of nanodiamond crystals with the presence of voids and 3D structure.

To further characterize this material, Raman spectroscopy ( $\lambda=325 \mathrm{~nm}$ and $514 \mathrm{~nm}$ ) was first carried out. As completely described in [10], we used both a Renishaw RM1000 and A Horiba Jobin Yvon T64000 spectrometer. The corresponding spectra are given in Figure 3Figure 3. Using the $514 \mathrm{~nm}$ excitation, spectra are dominated by a clear signature of disordered graphite, see the two maxima that correspond to the well-known " $D$ " and "G" lines peaking at about 1350 and $1590 \mathrm{~cm}^{-1}$ respectively. As sometimes observed in nanocrystalline CVD films, apparent maxima at about 1160 and $1480 \mathrm{~cm}^{-1}$ can also be distinguished. A careful examination of the signal further suggests the presence of a small component at about 1330 $\mathrm{cm}^{-1}$. Tuning the excitation wavelength at $325 \mathrm{~nm}$, this signal is now clearly observed, meaning that this material clearly contains diamond, along with some graphitic material. The diamond Raman mode is slightly downshifted to $1330 \mathrm{~cm}^{-1}$ with respect to bulk diamond $\left(1332 \mathrm{~cm}^{-1}\right)$. Moreover, its shape appears slightly asymmetric. A similar downshift and asymmetry can be observed by Raman on detonation nanodiamonds with size much smaller than $10 \mathrm{~nm}$ under the same detection conditions [8]. This was previously assigned to a 
confinement effect $[10,11]$. Thus, Raman spectroscopy suggests that this material is rather similar to detonation nanodiamond in terms of crystal size. However, the main difference between the present material and purified detonation nanodiamond is the higher relative amount of graphitic material. To get a more complete description of the porous diamond foam, HRTEM was used.

Those HRTEM investigations confirmed that this diamond material is composed of nanometric crystals with size included between 2 and $6 \mathrm{~nm}$ (Figure 4Figure 4a), which exhibit interplanar distances of $0.207 \mathrm{~nm}$ corresponding to diamond $\{111\}$ planes (Figure 4Figure $4 \mathrm{~b})$. FFT treatment of HRTEM images also reveals the presence of $\mathrm{sp}^{2}$ carbon with interplanar distances of $0.33 \mathrm{~nm}$ as shells surrounding diamond nanocrystals (Figure 4Figure 4c) which confirmed the Raman observations (disordered graphite with a small in plane coherence length). Moreover, HRTEM observations suggest that diamond crystals are coalesced forming a continuous nanocrystalline structure with a high specific surface area. The structure of the film consists of individual nanometric diamond particles connected to each other (Figure 5Figure 5). Nevertheless, it is quite difficult to separate individual crystals from HRTEM pictures which result from the superimposition of crystals (Figure 5Figure 5). This aggregation of diamond nanocrystals is easier to evidence after a short growth step (Supplementary Informations). The specific surface area measured on detonation nanodiamonds by BET is close to $350 \mathrm{~m}^{2} / \mathrm{g}$ [12]. This suggests porous diamond foam may behave a very high specific surface area.

BEN procedure was previously used to grow ultrananocrystalline (UNCD) diamond films in rich hydrogen/methane plasma (7\%) on silicon substrate [13]. HRTEM revealed the presence of diamond nanocrystals embedded in a graphitic matrix with size depending mainly on methane content and bias voltage [14]. This bulk material is very different compared to porous diamond foam composed of nanometric diamond grains exhibiting a high specific surface area (3D structure).

During BEN process, renucleation occurring on growing diamond crystals was observed on silicon substrates [15]. This phenomenon is related to the continuous ion bombardment of the surface. Such a mechanism may be also involved in the growth of porous diamond foam on iridium during BEN and may explain the presence of structural defects like dislocations observed by HRTEM. Compared to other substrates like silicon or $3 \mathrm{C}-\mathrm{SiC}$, the growth of diamond nuclei, i. e. the smallest diamond crystal thermodynamically stable [9], is prevented 
during the BEN process [8]. This peculiar behavior of iridium may explain the nanometric size of diamond crystals observed by HRTEM lower than $10 \mathrm{~nm}$.

To summarize, a porous diamond foam exhibiting nanometric diamond grain size (mean diameter $<10 \mathrm{~nm}$ ) was synthetized by prolonged Bias Enhanced Nucleation on iridium surfaces. This 3D material could be an excellent candidate for sensors applications due to its high specific surface area.

References:

[1] H. Masuda, M. Watanabe, K. Yasui, D. Tryk, T. Rao, A. Fujishima, Fabrication of a Nanostructured Diamond Honeycomb Film, Adv. Mater. 12 (2000) 444-447. http://doi.wiley.com/10.1002/(SICI)1521-4095(200003)12:6<444::AIDADMA444>3.0.CO;2-K (accessed November 3, 2014).

[2] T. Kondo, Y. Kodama, S. Ikezoe, K. Yajima, T. Aikawa, M. Yuasa, Porous borondoped diamond electrodes fabricated via two-step thermal treatment, Carbon N. Y. 77 (2014) 783-789. doi:10.1016/j.carbon.2014.05.082.

[3] H. Kato, J. Hees, R. Hoffmann, M. Wolfer, N. Yang, S. Yamasaki, et al., Diamond foam electrodes for electrochemical applications, Electrochem. Commun. 33 (2013) 88-91. doi:10.1016/j.elecom.2013.04.028.

[4] F. Gao, M.T. Wolfer, C.E. Nebel, Highly porous diamond foam as a thin-film microsupercapacitor material, Carbon N. Y. 80 (2014) 833-840. doi:10.1016/j.carbon.2014.09.007.

[5] S. Ruffinatto, H.A. Girard, F. Becher, J.-C. Arnault, D. Tromson, P. Bergonzo, Diamond porous membranes: A material toward analytical chemistry, Diam. Relat. Mater. 55 (2015) 123-130. doi:10.1016/j.diamond.2015.03.008.

[6] H. Zhuang, N. Yang, H. Fu, L. Zhang, C. Wang, N. Huang, et al., Diamond network: template-free fabrication and properties., ACS Appl. Mater. Interfaces. 7 (2015) 5384 90. doi:10.1021/am508851r.

[7] C. Hébert, J.P. Mazellier, E. Scorsone, M. Mermoux, P. Bergonzo, Boosting the electrochemical properties of diamond electrodes using carbon nanotube scaffolds, Carbon N. Y. 71 (2014) 27-33. doi:10.1016/j.carbon.2013.12.083.

[8] M. Schreck, J. Asmussen, S. Shikata, J.-C. Arnault, N. Fujimori, Large-area highquality single crystal diamond, MRS Bull. 39 (2014) 504-510. doi:10.1557/mrs.2014.96.

[9] N. Vaissiere, S. Saada, M. Bouttemy, a. Etcheberry, P. Bergonzo, J.C. Arnault, Heteroepitaxial diamond on iridium: New insights on domain formation, Diam. Relat. Mater. 36 (2013) 16-25. doi:10.1016/j.diamond.2013.03.010. 
[10] M. Mermoux, A. Crisci, T. Petit, H.A. Girard, J.-C. Arnault, Surface Modifications of Detonation Nanodiamonds Probed by Multiwavelength Raman Spectroscopy, J. Phys. Chem. C. 118 (2014) 23415-23425. doi:10.1021/jp507377z.

[11] S. Osswald, V. Mochalin, M. Havel, G. Yushin, Y. Gogotsi, Phonon confinement effects in the Raman spectrum of nanodiamond, Phys. Rev. B. 80 (2009) 075419. doi:10.1103/PhysRevB.80.075419.

[12] T. Petit, H.A. Girard, A. Trouvé, I. Batonneau-Gener, P. Bergonzo, J.-C. Arnault, Surface transfer doping can mediate both colloidal stability and self-assembly of nanodiamonds., Nanoscale. 5 (2013) 8958-62. doi:10.1039/c3nr02492j.

[13] Y.C. Chen, X.Y. Zhong, A.R. Konicek, D.S. Grierson, N.H. Tai, I.N. Lin, et al., Synthesis and characterization of smooth ultrananocrystalline diamond films via low pressure bias-enhanced nucleation and growth, Appl. Phys. Lett. 92 (2008) 133113. doi:10.1063/1.2838303.

[14] D. Pradhan, I.N. Lin, Grain-size-dependent diamond-nondiamond composite films: characterization and field-emission properties., ACS Appl. Mater. Interfaces. 1 (2009) 1444-50. doi:10.1021/am9001327.

[15] X. Jiang, W. Zhang, C.P. Klages, Effects of ion bombardment on the nucleation and growth of diamond films, Phys. Rev. B. 58 (1998) 7064.

doi:10.1103/PhysRevB.58.7064. 


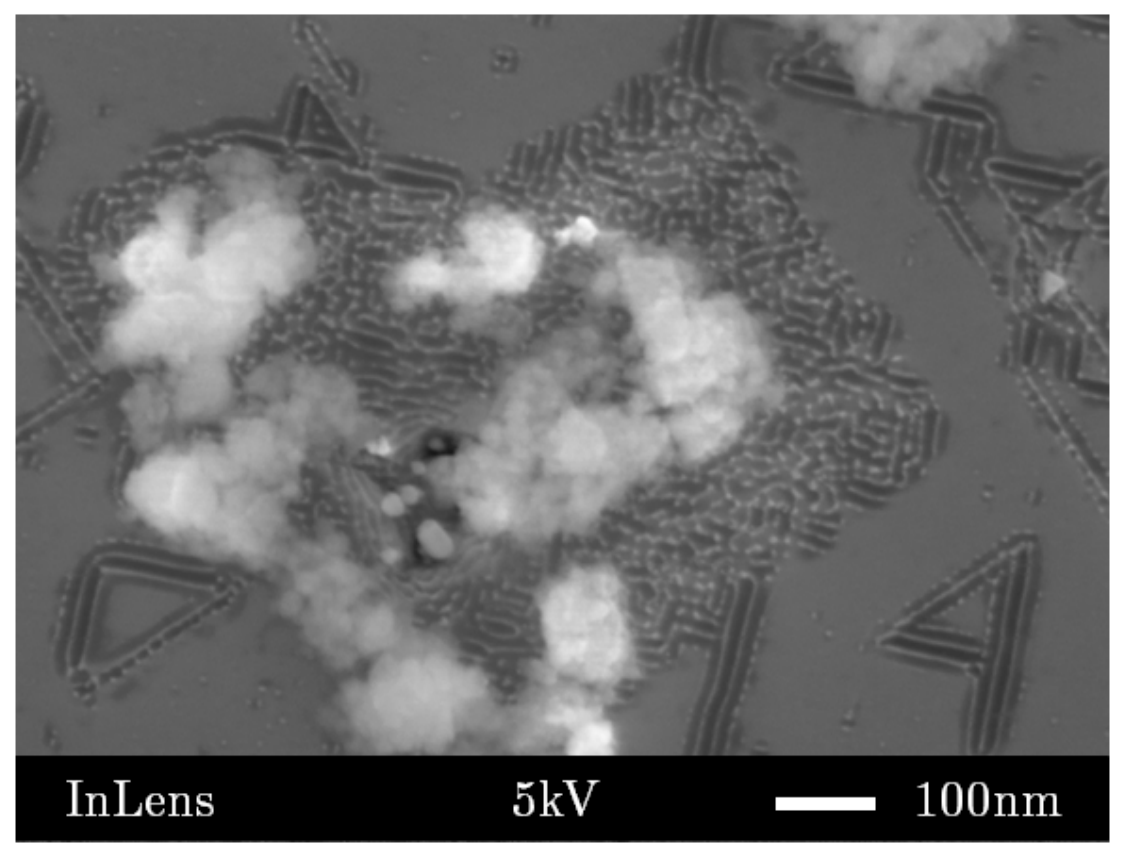

Figure 1 - FE-SEM pictures of diamond foam at the surface of $\mathrm{Ir} / \mathrm{SrTiO}_{3}$ sample after $20 \mathrm{~min}$ of BEN step. 

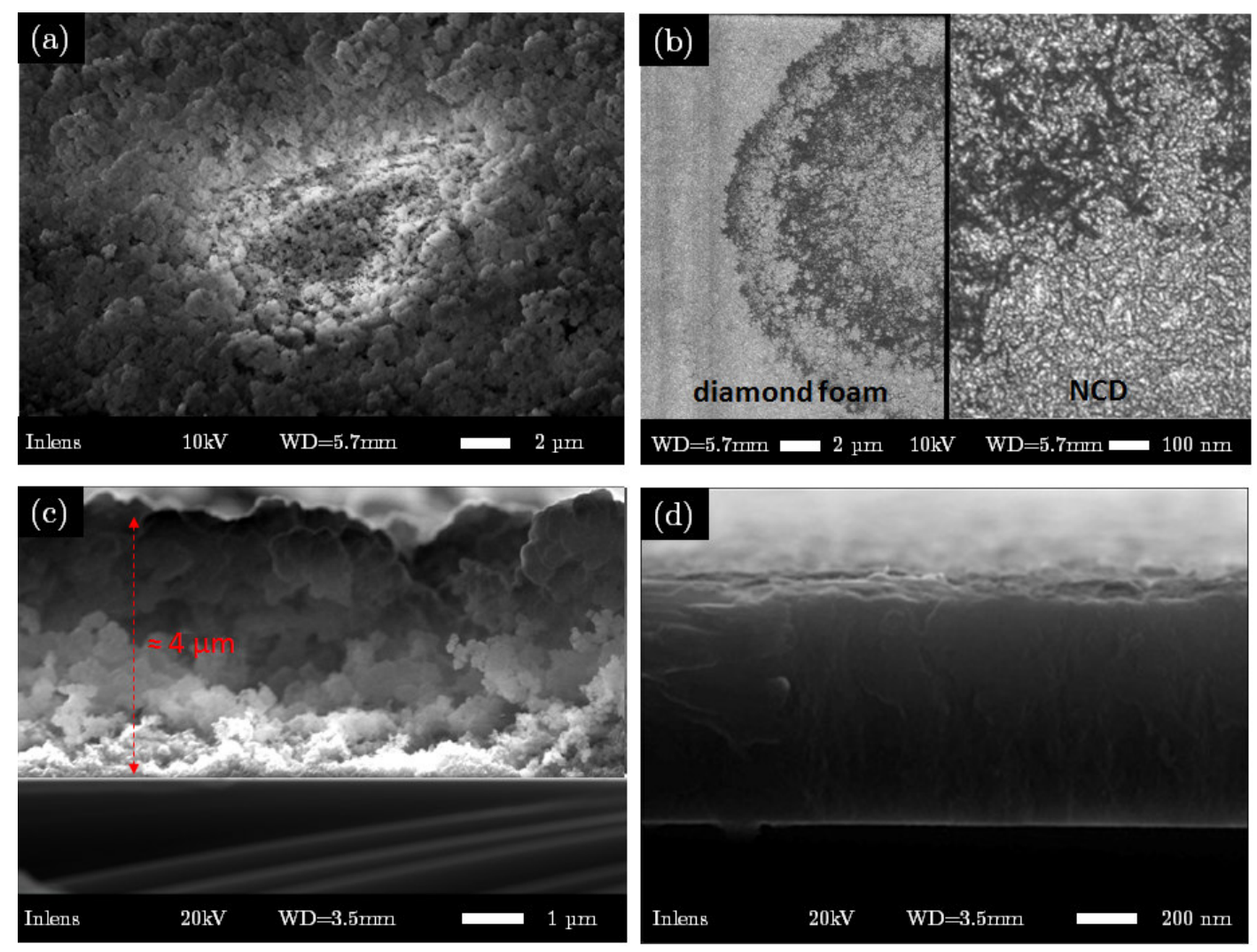

Figure 2 - FE-SEM pictures of the top view: a) diamond foam after 60 min of BEN plus micro-indentation, b) diamond foam and NanoCrystalline Diamond (NCD) after microindentation, c) cross section of diamond foam and d) cross section of NCD film. 


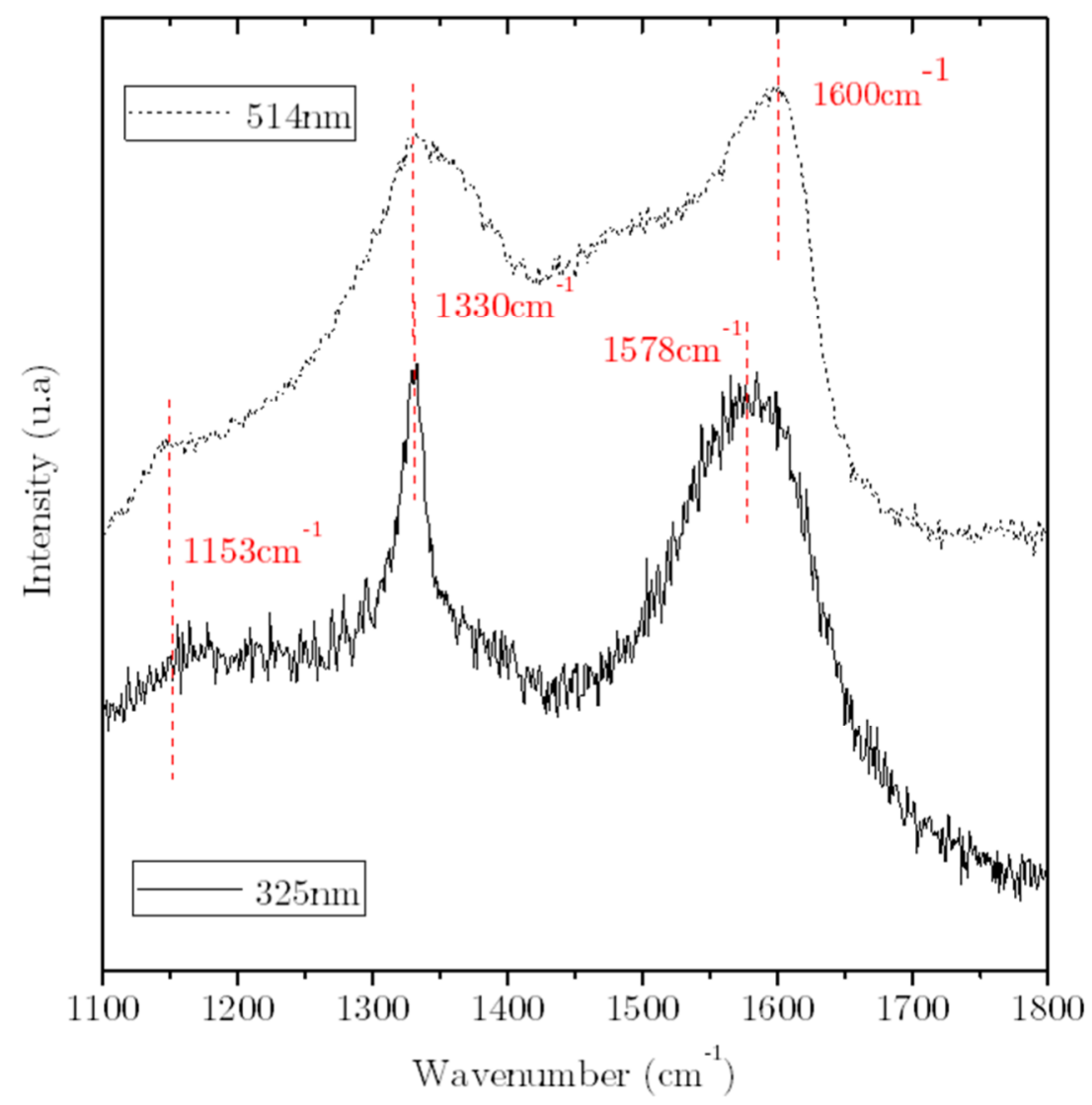

Figure 3 - Raman spectrum at $514 \mathrm{~nm}$ and $325 \mathrm{~nm}$ of the porous carbon material 


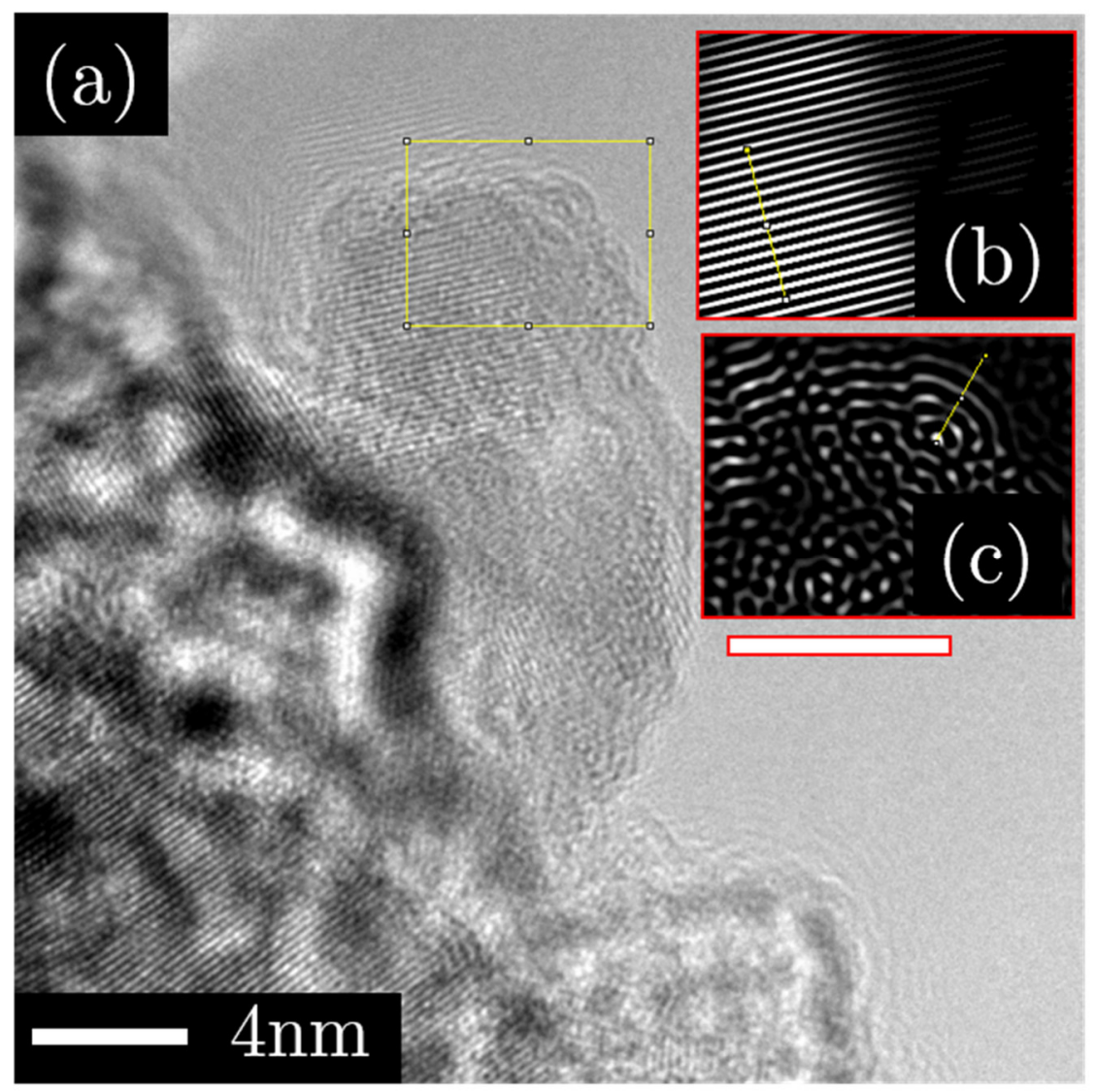

Figure 4 - (a) HRTEM picture of a diamond nanocrystal; FFT treated picture showing (b) diamond $\{111\}$ planes within the crystal (interplanar distance $0.207 \mathrm{~nm}$ ) and (c) $\mathrm{sp}^{2}$ carbon outer-shells (interplanar distance $0.33 \mathrm{~nm}$ ); Red scale bar $4 \mathrm{~nm}$. 


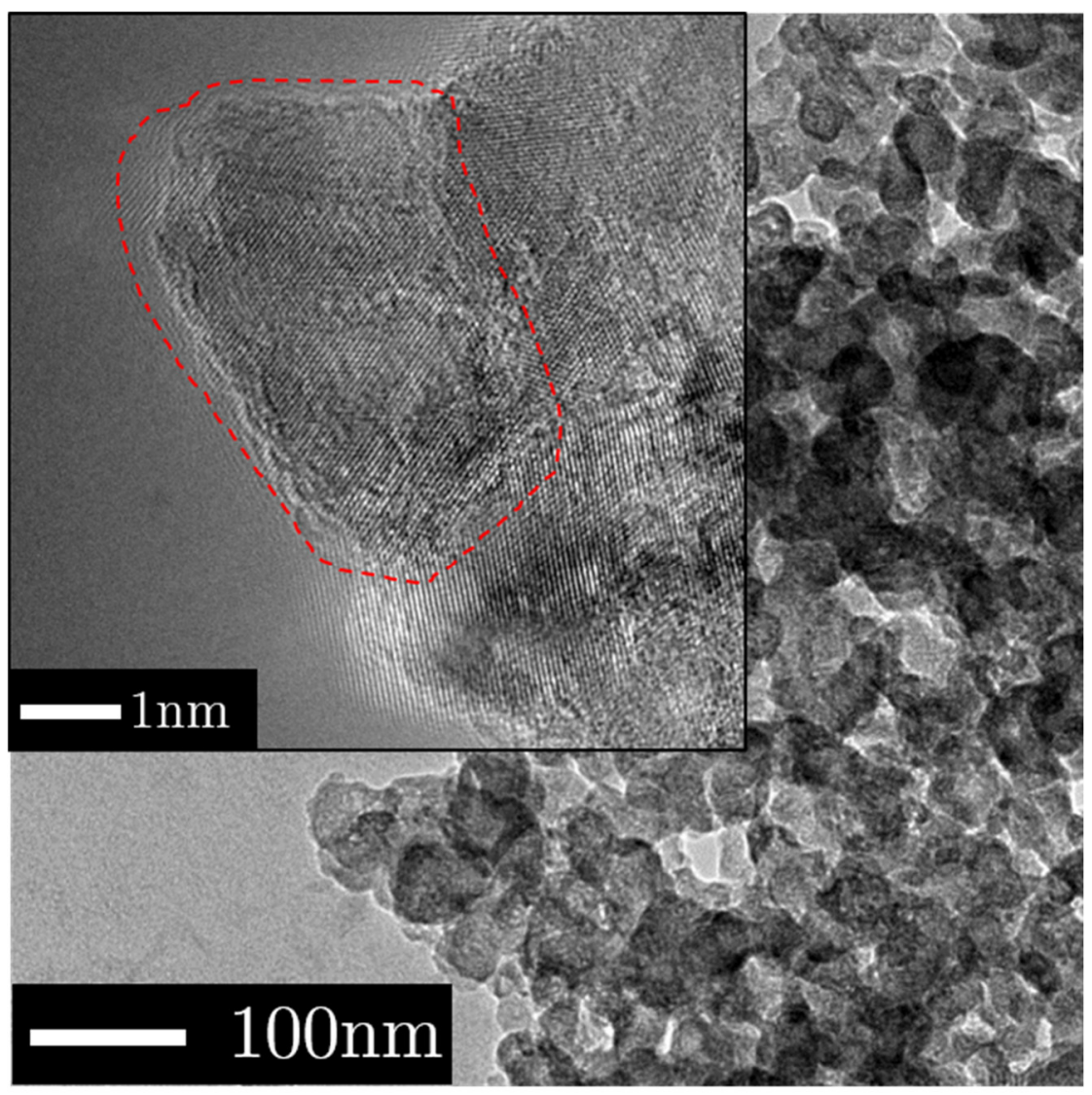

Figure 5 - HRTEM picture of diamond nanocrystals aggregated and in a frame, an aggregate marked out by the red line 


\section{Supplementary information}

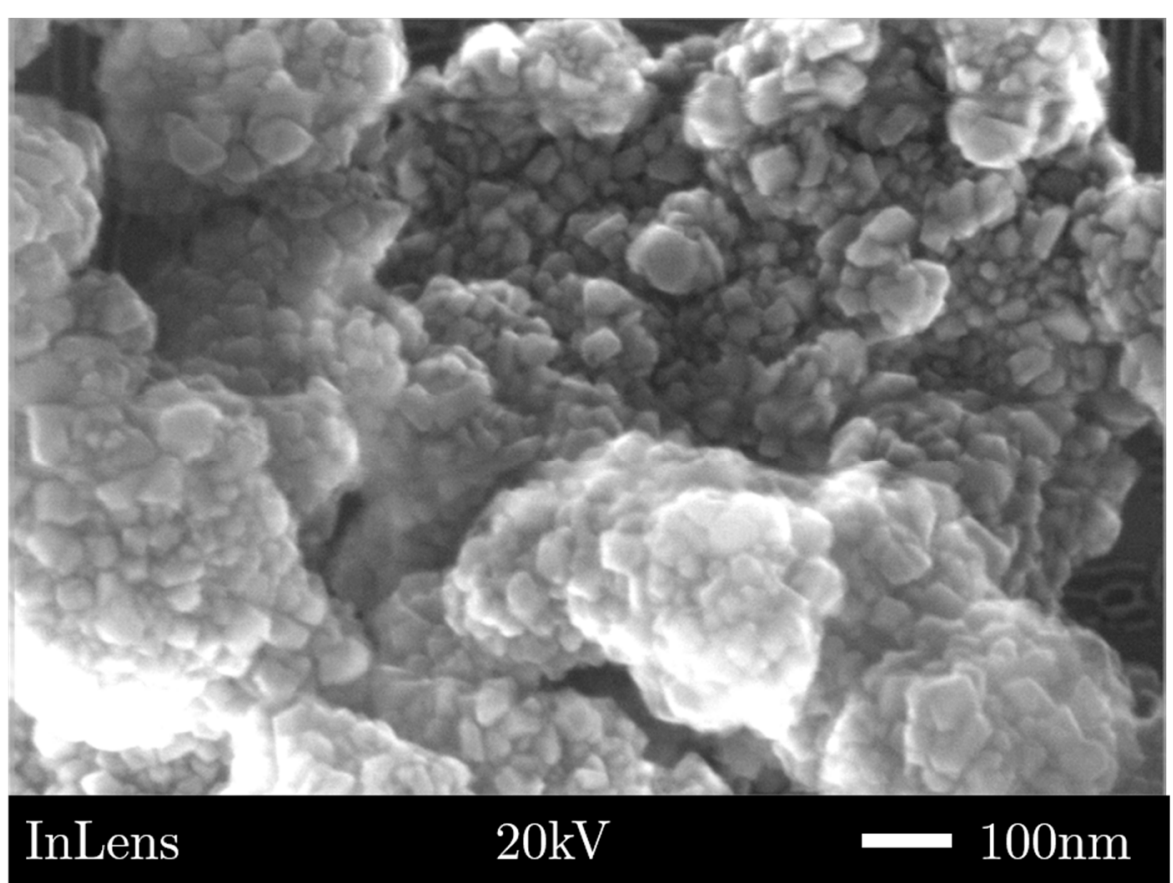

Figure 6-FE-SEM picture of diamond foam after a short growth step (growth conditions: $400 \mathrm{~W} / 20 \mathrm{mbar} / 0.6 \%$ of $\mathrm{CH}_{4}$ in $\mathrm{H}_{2} / 600{ }^{\circ} \mathrm{C} / 15$ minutes) 